\title{
LÉGITIMITÉ, LÉGITIMATION, NORMATIVITÉ
}

\begin{abstract}
André Berten ${ }^{1}$
L'usage sociologique des termes «légitimité» et «légitimation» propose une interprétation spécifique de la normativité inhérente aux comportements communicatifs et à la pragmatique du discours. Comme Bourdieu l'a défendu, l'attribution aux énoncés langagiers d'une performativité propre, d'une force intrinsèque, comme par exemple la force illocutoire, ne peut rendre compte des rapports sociaux sous-jacents aux échanges discursifs ${ }^{2}$. La pragmatique du discours, inspirée de Austin, Searle ou encore Brandom ${ }^{3}$, a néanmoins débouché sur une «théorie de l'agir communicationnel» qui se présente à la fois comme une théorie de la société à forte teneur sociologique, et une théorie du langage qui accorde à la logique du discours une certaine autonomie ${ }^{4}$. Je défendrai
\end{abstract}

1 André Berten est professeur émérite de la Faculté de philosophie de l'Université catholique de Louvain et professeur invité à l'Université Fédérale de Minas Gerais au Brésil.

2 BOURDIEU, Pierre (1982), Ce que parler veut dire: l'économie des échanges linguistiques, Paris, Fayard.

3 AUSTIN John Langshaw (1962), How to do Things with Words, Oxford, Oxford University Press; SEARLE John (1969), Speech Acts, Cambridge, Cambridge University Press; BRANDOM Robert B. (1994), Making It Explicit. Reasoning, Representing, and Discursive Commitment, Cambridge (Ma) \& London, Harvard University Press.

4 Cf. la distinction habermassienne entre «logique du développement» et «dynamique du développement», la première prétendant rendre compte des phénomènes de

Recherches en communication, $\mathrm{n}^{\circ} 25$ (2006). 
la thèse selon laquelle c'est dans la communication discursive que l'on peut trouver un certain type de fondement à la normativité et, par conséquent, aux formes de légitimité qui s'appuient sur cette normativité. Je rappellerai en premier lieu quelques aspects de l'approche sociologique de la question de la légitimité. Je présenterai ensuite une théorie de la normativité découlant des théories de l'action, dans une perspective cognitive, théorie qui permet de repenser la fonction des croyances dans la légitimité. Je montrerai enfin les implications du fait que la légitimité n'est pensable que dans le contexte langagier et, plus particulièrement, dans l'usage communicationnel du langage.

\section{Le point de vue sociologique}

Nous pouvons considérer que le concept de légitimation est essentiellement un concept sociologique visant à décrire un processus par lequel des individus sont amenés à reconnaître la légitimité du pouvoir, des institutions, des comportements, des usages, des conventions, des discours, etc.

Max Weber, dans une perspective de sociologie compréhensive, a donné une lecture possible de ces concepts :

«L'activité, et tout particulièrement l'activité sociale, et plus spécialement encore une relation sociale, peut s'orienter, du côté de ceux qui y participent, d'après la représentation de l'existence d'un ordre légitime. La chance que les choses se passent ainsi, nous l'appelons « validité »[Geltung] de l'ordre en question.» 1

Un ordre légitime est donc, du point de vue sociologique, une représentation, c'est-à-dire une «croyance». Quand la croyance est répandue, généralisée, c'est-à-dire quand elle engendre des comportements qui sont conformes à ce qu'elle impose normativement, l'ordre fonctionne, il est «valide» ou validé.

rationalisation du monde vécu, la seconde des contextes historiques contingents marqués, entre autres, par la pesanteur des rapports sociaux de domination.

1 WEBER Max (1971) Economie et société, tr. de l'allemand par J. Freund, P. Kamnitzer, P. Bertrand, E. de Dampierre, J. Mailard et J. Chavy, T.I, Paris, Plon, § 5, p.30 
Weber fait remarquer, ce qui me parait important, que l'obéissance à un ordre (et donc sa validité) ne peut être comprise, en général, uniquement par l'habitude ni par l'intérêt, mais implique aussi une représentation du «devoir» (selon les catégories de la rationalité en valeur), c'està-dire une croyance que l'ordre n'est pas valide seulement «parce qu'on a toujours fait comme ça» ou «parce que c'est mon intérêt individuel». En indiquant que, la plupart du temps, joue aussi une croyance dans la valeur de l'ordre, Weber introduit ce que l'on peut appeler une composante normative dans la structure de l'action. Comme il en reste à une perspective sociologique, il ne va pas plus loin dans son analyse de cette composante normative. Le fait d'ailleurs qu'il s'agisse de phénomènes qui sont présents «la plupart du temps» ou «en général» plaide pour une interprétation strictement sociologique des croyances, parmi lesquelles la croyance axiologique reste, tout compte fait, contingente. J'y reviendrai dans la deuxième partie de l'article.

Il est important, également, d'analyser ce qui, selon Weber, peut garantir un ordre légitime. La légitimité d'un ordre peut être garantie, pour les agents, soit de façon «purement intime» ${ }^{1}$, de façon affective, ou émotive; soit de façon rationnelle en valeur (d'ordre éthique, esthétique ou religieux); soit de façon «intéressée» (liée à un intérêt). Il est à remarquer que l'intérêt dont il est ici question n'est pas nécessairement l'intérêt économique ou utilitariste au sens étroit du terme. Nous avons intérêt également à respecter les conventions (parce qu'elles impliquent la réprobation des transgressions) ou le droit (qui implique contrainte et sanctions). D'un point de vue sociologique, la validité ou l'effectivité de l'ordre légitime se fonde sur des mécanismes sociaux. Ce sont eux qui assurent la domination exercée par un individu, un groupe ou une institution. On sait que Weber distingue quatre formes principales de motivations qui assurent simultanément l'obéissance aux ordres et la reconnaissance de leur légitimité : la tradition, les croyances au charisme d'une personnalité, les croyances rationnelles en valeur et les croyances en la légalité ou la rationalité des ordres².

1 Quand Weber parle de «intime», il ne se place pas au niveau simplement psychologique : les convictions intimes sont, bien entendu, largement déterminées par des croyances socialement diffusées ou acceptées, même si l'on peut concevoir des variations dans ces croyances, variations culturelles, historiques, et même individuelles (par exemple familiales)

2 Cf. WEBER, o.c., chap. III, «Les types de domination», pp. 219sq. 
La question que se pose Weber n'est donc pas de déterminer ce qui est légitime ou illégitime en soi, mais de décrire les mécanismes qui rendent une personne, une institution, une action légitimes du point de vue des agents. Certains interprètes ont estimé que Weber n'en restait pas à un point de vue purement descriptif, mais introduisait également un concept normatif, celui de rationalité. En effet, Weber établit une sorte de gradation dans les mécanismes cognitifs qui engendrent les croyances. Il considère que les individus qui accordent légitimité à quelque chose ou à quelqu'un pour des motifs strictement affectifs ou traditionnels ne manifestent aucune ou quasi aucune rationalité dans leur action, c'est-à-dire agissent de façon quasi automatique. Dans ce cas leur agir est dépourvu de «sens». Dès lors, le concept de rationalité, même s'il reste un concept descriptif, permet d'introduire une hiérarchie dans les types d'action. Est rationnelle l'action qui adopte les moyens adéquats aux fins, et qui fait preuve d'un jugement réflexif : qui discute des meilleurs moyens pour réaliser les buts que l'on s'est donnés, et compare les fins entre elles afin d'en évaluer les avantages respectifs' ${ }^{1}$. Ce faisant, elle introduit de fait un critère réflexif et normatif d'évaluation.

Si l'on s'intéressait maintenant aux sociologies d'inspiration marxienne ou critique - Marx lui-même, et aujourd'hui surtout Bourdieu et son école — on insisterait sur l'arbitraire fondamental de toutes les formes de légitimation et de légitimité en montrant comment elles sont avant tout reliées à l'exercice et à la justification du pouvoir. Or comme tout pouvoir exerce une violence, ne fût-ce que symbolique, la légitimation est toujours en quelque sorte «idéologique»².

Mon intention n'est pas d'entrer dans une discussion approfondie de la sociologie wébérienne ou marxienne. Simplement, nous voyons ici que les concepts de légitimité et de légitimation sont des concepts descriptifs et désignent des phénomènes qui doivent faire l'objet d'une

1 Que Weber pose à certains moments des jugements de valeur et adopte une attitude ambiguë vis-à-vis de la rationalité est manifeste. Mais cela n'enlève rien à l'intérêt de ses descriptions et de la compréhension de l'action humaine qu'elles offrent.

2 Encore une fois, s'il est évident que la force critique de ces analyses suppose bien des jugements de valeur, les thèses sur le fonctionnement idéologique des concepts de légitimation et de légitimité peuvent néanmoins être comprises comme des thèses strictement descriptives et explicatives des phénomènes sociaux. 
explication sociologique ou, à la rigueur, psychologique. Ces explications doivent nous aider à comprendre quels sont les mécanismes sousjacents à la reconnaissance de la légitimité des institutions ou du pouvoir. En d'autres termes, les processus de légitimation sont des processus «psychiques» qui engendrent des croyances dans la valeur normative des institutions (au sens le plus large du terme). Si l'on peut néanmoins parler ici d'une «sociologie», c'est parce que les croyances ne sont pas les purs produits d'une psychologie individuelle, mais le résultat de ce qui est dit, véhiculé, représenté au niveau social. En d'autres termes, ce sont des processus qui sont au cœur de la communication sociale, que celle-ci se déroule dans l'espace public, au sein des organisations, des institutions, des groupes formels ou informels. C'est pourquoi, une discipline (latu sensu) comme la sociologie de la communication est pleinement justifiée — pour ne pas dire... légitime.

$\mathrm{Ce}$ qui constitue, en quelque sorte, l'option méthodologique commune des lectures sociologiques des concepts de légitimité et de légitimation, c'est de ne pas se poser la question de savoir si, en fin de compte, il y a ou il n'y a pas de justification normative à ce qui est reconnu comme légitime, ou en d'autres termes, si l'on peut expliquer totalement - sans reste - les légitimités reconnues par les processus psychologiques et sociologiques (ou socio-psychologiques) de légitimation sans s'interroger sur les justifications des croyances en question ${ }^{1}$. Il ne faut, en effet, pas se cacher le fait que l'approche des sciences humaines implique une sorte de relativisme. La question est dès lors de savoir si la normativité — l'idée que certaines choses : institutions, personnes, discours, traditions, doivent faire l'objet de respect, de reconnaissance, d'obéissance — si cette idée a une quelconque justification.

Je voudrais introduire une perspective qui pourrait, éventuellement, indiquer quelques éléments de «normativité» non réductibles à l'approche sociologique.

1 Je n'entrerai pas ici dans la question épistémologique de la valeur de vérité que l'on peut attribuer aux croyances. Il s'agit d'une question complexe, non seulement du point de vue théorique (accès à la vérité ou à l'objectivité de nos connaissances), mais aussi des relations entre faits et valeurs, entre jugements théorique et jugements pratiques. 


\section{Du point de vue d'une théorie de l'action}

Je me situerai du point de vue justement des processus psychiques ou cognitifs qui sont en jeu dans les croyances à la légitimité d'un ordre social quelconque, d'une personne ou d'une institution.

Nous partirons de quelques éléments d'une théorie de l'action. L'action humaine - et dans doute déjà l'agir animal, au moins chez les animaux évolués - est un agir intentionnel, c'est-à-dire orienté vers des fins. Cela signifie que les comportements ne sont pas uniquement erratiques ou aléatoires, mais ne sont pas non plus exhaustivement déterminés par des causes que l'on pourrait insérer dans une théorie nomologique - comme le serait une théorie physique.

Or la structure même d'un comportement orienté implique le respect de certaines normes, normes instrumentales ou utilitaires, mais normes nécessaires pour le succès de l'action. Ces normes, bien entendu, ne sont pas pensées ou vécues comme normes. Nous pouvons seulement dire que, spontanément, nous savons comment nous comporter ou comment agir pour accomplir les actes quotidiens. Nous suivons constamment des «règles» qui sont simplement les conditions du succès de l'action en cours : des choses aussi simples que mettre un pied devant l'autre pour marcher, ouvrir la porte pour sortir, rompre le morceau de pain pour le mettre en bouche. Et des choses aussi complexes qu'élaborer une théorie sotériologique pour donner sens aux activités humaines.

Les limites entre ces règles apparemment «naturelles» et ce qui est culturel, ce qui résulte de normes non nécessaires, sont des limites absolument floues. Sans doute, il n'y a de portes et de pains que dans un milieu culturel donné. Mais sans doute aussi il y a une «naturalisation» des contraintes culturelles quotidiennes qui font qu'elles ne sont jamais, ou presque jamais, réfléchies comme normes, mais simplement vécues comme évidentes. Bourdieu a parfaitement décrit ces processus d'intériorisation ou de naturalisation des normes dans sa théorie de l'habitus'.

1 Les habitus, écrit-il, sont des «systèmes de dispositions durables et transposables, structures structurées prédisposées à fonctionner comme structures structurantes, c'est-à-dire en tant que principes générateurs et organisateurs de pratiques et de représentations qui peuvent être objectivement adaptées à leur but sans supposer la visée consciente de fins et la maîtrise expresse des opérations nécessaires pour les atteindre...» (BOURDIEU, Pierre (1980), Le sens pratique, Paris, Minuit, p. 88) 
Une question centrale ici est celle de la continuité/discontinuité entre d'une part ces normes quasi naturelles et ce que l'on pourrait appeler, en un sens très large, des règles morales. Je plaiderai, dans ce qui suit, pour une certaine forme de continuité, tout en acceptant que, rétrospectivement, il y a une grande différence entre le comportement humain (ou certains comportements humains) et le comportement animal. Ces différences sont liées à une compétence propre aux humains, leur capacité de réflexivité, c'est-à-dire de considérer les normes en tant que normes et donc d'être capables aussi de les abandonner, ou d'en changer si nécessaire ${ }^{1}$. Mais cette capacité présuppose justement que l'action est toujours déjà normée. Comme nous le verrons, la compétence réflexive est liée au langage, ce qui implique une complexité plus grande de la normativité propre à l'action.

Pour traiter de cette question difficile, je vais prendre un exemple - évoqué d'ailleurs, mais de façon un peu différente par Liénard ${ }^{2}$. Les théories de l'idéologie et les études de psychologie sociale ont en commun de nous montrer en quoi nos représentations et nos croyances peuvent être «fausses», biaisées, inadéquates ${ }^{3}$. Ce qui est présupposé dans l'un et l'autre cas c'est qu'il y a des croyances «vraies» et que nous devrions pouvoir, à partir d'une bonne théorie, corriger nos erreurs. Cette idée d'un certain rapport à la vérité a été critiquée comme étant encore métaphysique. Et il est vrai qu'une telle présupposition contient un aspect normatif, ne fût-ce que l'idée normative de vérité, qui nous permet de sélectionner entre le vrai et le faux. La question est alors

1 Comme le fait remarquer Philip Pettit: «Nous ne formons pas seulement des croyances et des désirs tout comme s'en montrent capables de nombreux animaux non-humains (...). Nous pouvons aussi exprimer de manière volontaire ou intentionnelle comment, à la lumière de nos croyances et de nos désirs, les choses se présentent. Nous n'avons pas que cette capacité stricte de croire que p, nous pouvons affirmer que p...» (PETTIT Philip (2001), «Deux sources de la moralité», Philosophiques, 28/1, printemps 2001, p. 178)

2 Dans cet ouvrage : «les catégorisations symboliques et les légitimations sont à la fois (a) des instruments de connaissance et de construction et de catégorisation du monde social ; (b) des moyens de communication ; (c) des moyens symboliques pour créer, maintenir ou déstructurer de la naturalisation, du contrôle, de la confiance, de la méfiance, de la justification. Bref, elles sont des instruments de pouvoir et/ou de domination symbolique.»

3 Cf. les travaux pionniers réunis par KAHNEMAN, D., SLOVIC, P., \& TVERSKY, A. (eds) (1982), Judgement under uncertainty: Heuristics and biases, Cambridge, Cambridge University Press. 
de savoir si nous pouvons nous passer complètement d'une telle référence.

Ce que nous montrent, en tout cas, la psychologie cognitive et les recherches de psychologie sociale qui intègrent des perspectives cognitives, c'est que notre connaissance du réel procède de formes de catégorisation qui sont absolument nécessaires pour que le monde soit minimalement compris et utilisé. Non seulement le langage est un puissant instrument de mise en ordre du monde, de conceptualisation, mais, en deçà du langage, ou parallèlement, nos «modèles mentaux» ${ }^{1}$ sont, à l'instar des catégorisations linguistiques, des conditions de possibilité de toute connaissance. S'il en est ainsi, il faut reconnaître que cette approche du monde conditionne la possibilité même de sélection et d'évaluation de ce qui est ou non adéquat, pertinent, satisfaisant.

Or, en constatant cela, nous devons accepter que les catégories, concepts, termes, que nous utilisons ont un aspect normatif. Ce sont des règles d'interprétation du monde que nous devons suivre, et ce sont aussi des manières - relativement arbitraires — d'imposer un ordre du monde. Ce sont ce que Kant appelait des impératifs hypothétiques. Nous devons les suivre si..., si nous voulons réussir dans nos actions, si nous voulons éviter les menaces de l'environnement, si nous voulons coordonner nos actions avec autrui. Mais peut-être aussi, si nous voulons être heureux, si nous voulons être moraux. Et nous imposons un ordre au monde, car les obligations cognitives ne sont pas des nécessités : il y a un certain arbitraire dans les choix, une certaine contingence dans la détermination des chemins que nous suivons.

Cela signifie que nous ne pouvons pas, personne ne peut, vivre sans se soumettre à une normativité. Ce qui caractérise l'humanité - et nous différencie des animaux — c'est notre capacité réflexive : la capacité de réfléchir sur les normes et, éventuellement, de les remettre en question. Cette capacité présuppose un rapport à un ordre normatif qui, certes, n'est pas absolu ni totalement contraignant, mais reste toujours présent. Si nous pouvons toujours, localement ou ponctuellement, nous dérober aux exigences normatives, quelles qu'elles soient, nous ne pouvons pas nous soustraire à ce que l'on peut appeler «l'ordre normatif» en tant que tel ${ }^{2}$. Il constitue ce que Husserl a appelé un horizon et ce que

1 Cf. par exemple JOHNSON-LAIRD Philip .N., Mental Models. Towards a Cognitive science of Language, Inference, and Consciousmess, Cambridge, Cambridge University Press, 1983

2 De même que nous pouvons thématiser et remettre en question certains aspects du 
Habermas considère comme le monde vécu. Sans aucune référence à ce qui convient, ce qui est préférable, ce qui est obligatoire, ce qu'il vaut mieux de faire, nous serions des zombies déboussolés.

Ajoutons que, comme l'ordre normatif est un ordre social, il se constitue dans l'intersubjectivité ou, si l'on préfère, dans les relations sociales en général. Il appartient à l'agir communicationnel, à la pragmatique des relations sociales, au monde des négociations, des délibérations, des discussions.

\section{De la légitimité démocratique}

Revenons maintenant à la question de la légitimité.

Un ordre légitime appartient à l'ordre normatif en général. Mais les ordres reconnus comme légitimes, tels qu'ils existent empiriquement, sont des phénomènes historiques et sociaux. L'ordre qui semble incarner aujourd'hui la légitimité politique est l'ordre démocratique. Cela ne signifie pas qu'il soit valide universellement (au sens de Weber : que sa reconnaissance soit effective partout et dans toute culture), mais il jouit d'une forte légitimité dans la plupart des pays occidentaux et peut-être d'une légitimité potentielle ailleurs. Ceci bien entendu n'est encore qu'une constatation empirique, descriptive.

De ce point de vue descriptif, une caractéristique essentielle des démocraties contemporaines est de «produire» les normes de légitimité1. Cela signifie à la fois leur refuser un fondement absolu, extérieur, transcendant, et, en se considérant comme l'auteur des normes, se donner également le droit de les changer, pourvu que cela se fasse selon les règles procédurales des États de droit. Cela n'est possible, toutefois, que parce que - et dans la mesure où - est reconnue la légitimité de la procédure. Ces normes procédurales sont, ou ont été, idéalement produites à partir de débats parlementaires qui eux-mêmes reflétaient ou reflètent l'état de l'opinion publique. Celle-ci ne doit pas être conçue comme «consensuelle», mais traversée de conflits et de relations de pouvoir. Mais l'établissement de la procédure présuppose que la discussion — ou pour le moins la violence symbolique — se soit

«monde vécu», mais jamais le monde vécu dans son ensemble qui reste, selon les termes de Husserl, un horizon indépassable.

1 Comme Alain Touraine avait, en son temps, parlé de «produire la société», c'està-dire réincorporer dans le corps social la genèse de l'ordre normatif (TOURAINE Alain (1973), Production de la société, Paris, Seuil) 
substituée à la violence brute ou à la force, comme moyen de régler les conflits et que cette substitution soit revêtue d'une certaine légitimité. Cette reconnaissance est une reconnaissance sociale et exclut que la légitimité puisse être attribuée à une volonté individuelle et arbitraire revendiquant pour elle-même des normes particulières — du moins en principe. Nous avons ainsi un double niveau de légitimité : nous considérons comme légitimes les normes adoptées démocratiquement; mais, plus profondément, nous devons considérer comme légitime la procédure qui produit ces normes ${ }^{1}$.

Une double question surgit ici.

En premier lieu, la question empirique est de savoir si ceux qui accordent légitimité aux procédures démocratiques le font parce qu'ils attribuent à ces procédures une valeur absolue, ou du moins parce qu'ils attribuent, globalement, une qualification positive indiscutable à la démocratie. En d'autres termes, les régimes démocratiques libéraux représenteraient dans ce cas «l'axe du bien» et seraient investis de la grandeur et du prestige de ce qui vaut par soi-même. Leur légitimité aurait alors, selon les termes de Weber, une validité effective. Mais, bien entendu, la croyance dans le caractère absolu de la valeur des principes démocratiques ne devrait être considérée que comme un fait, psychologique ou sociologique, et ne signifierait pas un privilège en soi ou une fondation indiscutable.

La seconde question, toujours dans cet espace des descriptions empiriques, renvoie à la diversité des croyances. La thèse de la forte légitimité des principes démocratiques et de leur universalité potentielle n'est pas évidente. De nombreux critiques de la démocratie - de droite ou de gauche - ont soutenu des thèses traditionalistes, autoritaires, aristocratiques, technocratiques, élitistes, anarchistes. Et, par ailleurs, les fondamentalismes, intégrismes et théocraties de tout genre n'accordent de légitimité qu'à des puissances surhumaines ou transcendantes. Rien ne garantit donc que les thèses démocratiques s'imposeront à l'avenir.

1 C'est ce que soutient, par exemple, Claude Lefort : «... à la notion d'un régime réglé par des lois, d'un pouvoir légitime, la démocratie moderne nous invite à substituer celle d'un régime fondé sur la légitimité d'un débat sur le légitime et l'illégitime débat nécessairement sans garant et sans terme.» (LEFORT Claude, (1986), Essais sur le politique XIX ${ }^{e}-X X^{e}$ siècles, Paris, Seuil, p. 53) 


\section{Légitimité et langage}

Les thèses antidémocratiques attribuent une légitimité et donc une normativité à des entités souvent personnalisées (divines ou sacrées) et à des principes qui sont censés avoir été décidés par ces entités. Nous pouvons donc soutenir que, même dans les cas les plus extrêmes, il y a une normativité, et que cette normativité est «morale», au sens sociologique du terme. De plus, les normes en question sont en général formulées dans des codes, des injonctions. Elles peuvent aussi être implicites, suivies sans être thématisées et donc sans être jamais problématisées. Mais s'il s'avère nécessaire de justifier les comportements et les croyances, il faudra expliciter les normes effectivement acceptées - ce à quoi, dans des dimensions différentes, se consacrent la théologie, l'apologétique, l'herméneutique et, dans un monde sécularisé, les déclarations des droits de l'homme ou les principes constitutionnels, et de façon plus générale, les codes juridiques.

Cette justification des normes, quel que soit son degré de réflexivité, ne peut se faire que par le langage. Or, comme nous l'avons signalé à propos de la catégorisation, le langage est intrinsèquement normatif. Mais cet aspect normatif n'est pas suffisant pour accorder une légitimité autre que celle décrite sociologiquement, c'est-à-dire une légitimité relative. Il faut encore s'interroger sur l'usage ou les usages du langage.

C'est dans l'exploration de l'usage pragmatique du langage qu' ont travaillé une série de penseurs contemporains qui, depuis Wittgenstein jusqu'à Habermas et Brandom, montrent ou tentent de montrer qu'un usage discursif libre, non limité autoritairement par une entité normative, a - c'est du moins une hypothèse assez bien confortée — certaines propriétés, par exemple de ne pas accepter n'importe quel type de justification. La force de l'argument de Habermas est ainsi de ne pas renvoyer la question de la légitimité aux propriétés psychologiques des sujets, à leur tendance à attribuer une autorité à des personnes ou des institutions, et donc de ne pas se fonder sur la bonne volonté des individus, mais bien sur les contraintes du discours communicatif. C'est pourquoi une «théorie de l'agir communicatif» peut être comprise à la fois d'un point de vue sociologique comme le produit d'une société où les conflits sociaux auraient tendance à se traduire symboliquement dans l'espace du discours et comme l'armature théorique présupposée par une sociologie qui ne peut faire l'impasse sur le fait que les rapports sociaux sont aussi - en général et la plupart du temps dirait Weber - 
des rapports langagiers. Ce qui d'ailleurs met directement en évidence le paradoxe maintes fois relevé du statut du discours sociologique luimême comme participant des échanges langagiers, c'est-à-dire des rapports sociaux, mais prétendant en même temps garantir la rationalité de son propre propos. De cette pragmatique résulte, selon moi, que les analyses sociologiques des formes de communication dans les organisations, les entreprises, les institutions de médiation, les administrations tout comme l'analyse de la fonction des «communicateurs», ne peuvent échapper à cette pragmatique de la communication et sont parties prenantes, de façon ou d'autre, des discussions qu'elles se proposent d'investiguer. Or, ce faisant, elles sont soumises aux contraintes du langage, contraintes qui ne sont pas différentes de celles qui régissent les objets analysés.

Un des arguments en faveur d'une certaine autonomie du langage concerne les phénomènes d'abstraction et de généralisation impliqués par la nature conceptuelle du langage. Les apprentissages individuels et collectifs se font à travers des échanges linguistiques qui impliquent des décentrements et des généralisations. C'est ainsi par exemple que Piaget et Kohlberg ont analysé le développement moral à partir des apprentissages cognitifs et des capacités d'universalisation du jugement. Mais ces formes de généralisation peuvent aussi s'appuyer sur l'observation, par exemple sur les contraintes expérimentales dans les débats scientifiques.

À travers le langage, le concept de légitimité est ainsi amené à rencontrer des règles universalisables ou généralisables. Il reçoit, diton, un fondement rationnel quand cette généralité est thématisée ou devient réflexive. Un des arguments essentiels de la pragmatique du discours consiste à montrer que cette contrainte à la généralisation place l'interlocuteur devant un dilemme : ou bien entrer dans la logique argumentative, ce qui implique inter-locution et donc décentrement, ou bien renoncer à la discussion et se réfugier dans ce que Éric Weil a qualifié de «violence» ${ }^{1}$, celle de la conviction intime, de la pure subjectivité ou du fanatisme aveugle des certitudes transcendantes.

A partir de ces considérations théoriques, je voudrais revenir sur les thèses d'inspiration critiques, c'est-à-dire les thèses qui, comme le montrait la citation de Liénard en note, passent rapidement de la fonc-

1 WEIL Eric (1950), Logique de la philosophie, Paris, Vrin. 
tion cognitive des catégorisations à une fonction de domination. Si l'on accepte la thèse cognitive, les légitimations sont des processus psychiques très généraux, inévitables du point de vue de notre rapport théorique et pratique au monde, et dont on ne peut déduire une thèse générale qui les subordonneraient exclusivement aux phénomènes sociaux de domination. Comment donc intégrer un point de vue sociologique dans l'analyse des formes réelles de légitimation? Une piste féconde consiste à distinguer entre pragmatique et sémantique du langage. $\mathrm{Si}$, par exemple, toutes les langues utilisent et doivent utiliser des concepts ou des catégories, si elles ont toutes une syntaxe, il est vrai aussi que l'incarnation des compétences cognitives dans des langues concrètes implique nécessairement des choix. De la même façon que la psychologie sociale cognitive montre clairement que les catégorisations entraîne, généralement et la plupart du temps, des «stéréotypes», les représentations du monde peuvent facilement devenir des «idéologies».

À mon avis, le point le plus difficile est le suivant. La thèse de la continuité implique qu'il est très difficile, sinon impossible, de tracer une ligne de séparation - une coupure épistémologique — entre ce qui relève des mécanismes cognitifs indispensables au simple fonctionnement des sociétés d'une part, et ce qui relève d'une forme de domination et de ses justifications. Nous devons en effet admettre que l'évolution nous a doté de compétences cognitives utiles à la fois à notre survie personnelle et à celle des groupes dont nous faisons partie (sinon à celle de l'espèce en tant que telle). Mais il est également vrai que les connexions entre savoir et pouvoir font partie depuis toujours de l'histoire de l'humanité. Il est très difficile, dans cette perspective, de départager entre des prestations cognitives «positives» et «négatives», «fonctionnelles» et «injustes».

Le vieux débat entre les sociologies fonctionnalistes et les sociologies critiques repose sur cette distinction. Les sociologies fonctionnalistes sont plutôt intéressées aux conditions nécessaires à la cohésion, à la perpétuation de l'organisation sociale. Les sociologies critiques sont au contraire sensibles aux contradictions sociales, aux phénomènes de domination, de répression, d'exploitation, c'est-à-dire à ce que l'on peut considérer comme des particularisations instrumentales du savoir.

Or, si l'on admet que la normativité est une caractéristique structurelle des opérations cognitives en général, la distinction entre une normativité «légitime» et «illégitime»n'est jamais tranchée. Toute relation sociale implique un jeu pragmatique de relations dans lesquelles le locuteur tient pour légitimes ses énoncés et les propose avec plus ou 
moins de force à la reconnaissance d'autrui. Bien entendu, la force de l'énoncé tient aussi à l'état des relations sociales, économiques, institutionnelles. Mais ce point de vue «externe» dépend d'un jugement politique et non du fait que certaines légitimations seraient «justes» tandis que d'autres seraient le reflet ou la rationalisation de positions de puissance; ou que certaines formes de légitimité pourraient être argumentées rationnellement tandis que d'autres seraient purement idéologiques.

Le seule espérance que l'on peut avoir est que le recours à une procédure démocratique - une procédure permettant au maximum la participation égalitaire de tous - sera plus à même de neutraliser les effets d'unilatéralité liés aux situations sociales existantes. Si l'on accorde de plus un certain crédit à «l'éthique de la discussion», c'està-dire aux vertus contraignantes du discours, du langage et de sa pragmatique, on peut suggérer que le modèle de démocratie délibérative serait le plus à même d'établir les conditions d'une légitimité, non pas incontestable, mais dont les formes de contestation relèveraient de la dispersion maximale des effets normatifs liés à tout énoncé langagier. Le modèle de démocratie délibérative, malgré toutes ses limites internes, n'est pas exclusivement un modèle politique. Les formes de discussion dans les institutions, les organisations, les administrations, les groupes en général, quand elles font appel à ce qui est légitime, juste, justifié, correct, marquent sans aucun doute des positions de pouvoir et cherchent à les légitimer. Le problème est que les arguments, une fois rendus publics, acquièrent une force intrinsèque que les protagonistes d'un conflit peuvent s'approprier parce qu'ils correspondent à ce qui peut être justifié, c'est-à-dire, dans le meilleur des cas, à ce qu'aucun argument acceptable n'est susceptible de démentir. 